\title{
Report
}

\section{How Many Embryos Should Be Transplanted After IVF Having Previous Pre-Eclampsia: A Case Report}

\author{
Min Han, Xuelan Li \\ The First Affiliated Hospital of Xi'an Jiaotong Medical University, Xi'an, China \\ Email address: \\ shandawar@126.com (Min Han), lixuelan1225@126.com (XueLan Li)
}

\section{To cite this article:}

Min Han, Xuelan Li. How Many Embryos Should Be Transplanted After IVF Having Previous Pre-Eclampsia: A Case Report. Journal of Gynecology and Obstetrics. Vol. 6, No. 4, 2018, pp. 80-85. doi: 10.11648/j.jgo.20180604.12

Received: July 7, 2018; Accepted: July 26, 2018; Published: August 16, 2018

\begin{abstract}
Pre-eclampsia is a pregnancy-induced hypertensive disorder which affect multiple organ functions, it is one of the main factors increasing the morbidity and mortality of the maternal and neonatal. Furthermore, with the increasing rate of infertility or sub-fertility, more and more couples are conceiving assisted reproductive therapies, especially in vitro fertilization (IVF), which brings high possibility of multiple pregnancy due to its unique process and the patients demands. As is well known that multiple pregnancy may face great challenges of complications such as pre-eclampsia, gestational diabetes, intrahepatic cholestasis of pregnancy etc. If pre-eclampsia is not treated and monitored very well on time the mothers perhaps will have eclampsia, placental abruption or multiple organ dysfunction leading to death, likewise those fetus whose mother suffered from Pre-eclampsia also face the risks of fetus growth restriction, fetal stress or even stillbirth. We represent a patient in our clinic who had done twice in vitro fertilization-embryo transplantation(IVF-ET) with several high risks of pre-eclampsia and followed by recurrent Pre-eclampsia and Intrahepatic Cholestasis of Pregnancy, we fully recognize her risks in her second pregnancy and give a close antenatal surveillance, and she finally have two alive babies, but a large amount of money was spend for the two preterm babies in hospitalization in NICU and also the mother herself became exhausted and almost lost her hope. It reminds us that before conceiving IVF technology for patients, the indication must be clear, the adverse effect must be brought into consideration, cooperation must be obtained from both the reproductive clinicians and obstetrician to fully balance the benefit and adverse effect, attention must be paid to choose the best method during the assisted reproductive therapies and acquire the utmost benefit and avoid the side effect as possible.
\end{abstract}

Keywords: Pre-eclampsia, IVF-ET, Intrahepatic Cholestasis of Pregnancy

\section{Introduction}

Pre-eclampsia is clinically defined by hypertension and proteinuria, with or without pathological edema that occurs after 20 weeks' gestation, but can also present up to 4-6 weeks post-partum [1]. Worldwide, preeclampsia and eclampsia is responsible for approximately 14 percent of maternal deaths per year [2]. Early diagnosis and appropriate management is extremely important in patients with pre-eclampsia or those who has high risks of pre-eclampsia. The exact etiology is unknown but some high risks are recognized worldwide, such as prior pre-eclampsia, chronic hypertension, multiple gestation, pre-gestational diabetes, maternal BMI $>30 \mathrm{~kg} / \mathrm{m}^{2}$, anti-phospholipid syndrome/SLE
[3]. However, with successfully and widely using of assisted reproductive technology(ART) for infertility, small studies have also suggested an association between the use of IVF and birth defects, adverse neurodevelopmental outcomes, pre-eclampsia, prenatal mortality, placenta previa, and an increased rate of cesarean delivery. Patients using IVF were 2.7 times more likely to develop pre-eclampsia [4]. Moreover, one of the most common complications related to the IVF technology is the multiple pregnancy. A multiple pregnancy is a risk factor for hypertensive disorders in pregnancy [5]. Multiple pregnancy often increase uterine pressure to an unusually high level, which cause severe placental ischemia and hypoxia, resulting in vascular endothelial cell damage and dysfunction [6]. Not only multiple pregnancy, but the 
assisted therapy method also play an important role in the maternal and neonatal outcome, following the ICSI-ET(intro-cytoplasmic sperm injection and embryo transplantation) process, the invasive procedure can create some unfavorable condition that may impact the implantation of the embryos and change the endometrial microenvironment, which can affect the health of the off springs [7].

\section{Case Presentation}

A 28 years old woman, at 23 weeks gestation visited the obstetric clinic complaining edema of lower limbs for 20 days. 4 days ago she had her routine pregnancy check-up, with a blood pressure of $140-155 / 99-100 \mathrm{mmHg}$, measured twice in local clinic in resting state, and $3+$ protein on urine dipstick, she also represented abnormal vaginal bleeding without any abdominal pain for half day. Personal history: she had an abnormal menstrual cycle (7/30-90) since her menarche at 17 years old, she was diagnosed as Polycystic Ovarian Syndrome (PCOS) 6 years ago, she had ectopic pregnancy 3 years ago and had Laparoscopic salpingectomy. Assistant Reproductive Technique (ART) was performed for her pregnancy this time because of infertility for 3years, she took dydrogesterone orally and progesterone injection at her first trimester of pregnancy after IVF-ET. No smoking and alcohol or drug abuse history, her mother passed away because of hypertension, no special inherited disease or similar case was found in her family. After admission to our hospital, lab tests show: proteinuria $3 \mathrm{~g} / 24 \mathrm{~h}$, her liver function tests reveal hypoproteinemia (Serumalbumin was 26g/l), serum urea, creatinine, hemoglobin, platelet, coagulation test and autoimmune antibody tests were all normal. ultrsonography showed fetal death, maternal ascites, and pelvic effusion. electrocardiography revealed Pericardial effusion, left ventricle hypertrophy along with mild pulmonary hypertension (pulmonary artery systolic pressure was $40 \mathrm{mmHg}$ ), left ventricle Ejection Fraction (EF) was 55\%, ultrsonography of liver, kidney, ureteral and bladder were normal, fundus examination indicated Retinal arteriosclerosis Ultimate diagnosis: pre-eclampsia (severe type), 23 weeks pregnant, Pericardial effusion, stillbirth. $30 \mathrm{mg}$ of oral nifedipine and $100 \mathrm{mg}$ intravenous labetalol was administered antenatally as soon as she was admitted, besides, supplementation of albumin and the diuretic drugs were also added immediately. After two days she gave birth through vaginal delivery to a dead male weighting $580 \mathrm{~g}$ with the help of induction by injecting rivanol into amniotic cavity. The fourth day after deliver, her blood pressure was normal and proteinuria arrived to $6.7 \mathrm{~g} / 24 \mathrm{~h}$, she was discharged and advised to have close follow-up in Cardiac and Nephrology clinic. She couldn't recalled when her proteinuria turned to normal.

The same patient came to clinic after 3 years at $6^{\text {th }}$ week gestation for regular antenatal check up. she had her blood pressure measured weekly and deny any abnormality of blood pressure since her last labor and she also had a good preconception counsel and physical examination in order to have a higher rate of a living child. All the preconception lab tests were good, she strongly requested the doctor to transfer two embryos during IVF well knowing possible risks and complication of having twins and ICSI method was applied to her later. She visited the obstetrics high-risk clinics as soon as she was informed of two living embryos at $6^{\text {th }}$ weeks of gestation, considering of her pre-eclampsia history, she was recommended to have her blood pressure measured twice daily and visit the clinic every 2 weeks, she looked very anxious recalling her unfortunate pregnancy history, so we arranged for her psychological counseling and encourage her to have a positive idea that people having previous pre-eclampsia history can also have a successful following pregnancy, at the same time we also informed her family members the high risks and encouraged them to avoid any form of fight or depression on her, making sure she has a relax environment and had as less anxious as possible. As medicine intervention of recurrent pre-eclampsia, Low molecular weight heparin, aspirin $(100 \mathrm{mg} / \mathrm{d})$, multiple vitamins, calcium were given as pre-eclampsia prophylaxis from $12^{\text {th }}$ week of gestation. Since given injection of low molecular weight heparin, monthly coagulation test was arranged in the first and second trimester of pregnancy and result seemed to be good. she remained symptom free and a good BP control before 22 weeks within the range of $106-118 / 68-83 \mathrm{mmHg}$ (Figure 1). At her $26^{\text {th }}$ week gestation, her lower limbs edema $(+)$ appeared, diastolic blood pressure increased to $80-90 \mathrm{mmHg}$ with a normal systolic blood pressure. Considering that rapidly increase of blood volume in the third trimester and it's threaten to cardiovascular system possibly will increase her blood pressure, we gave oral methyldopa $250 \mathrm{mg}, 12$ hours apart to reduce the likelihood of developing severe maternal hypertension and other complications such as low platelets and elevated liver enzyme with symptoms. Her blood pressure remained to be normal, but her serum albumin decrease to $30 \mathrm{~g} / \mathrm{l}$ with normal liver enzymes in her $28^{\text {th }}$ week of pregnancy. and almost had no weight gain in 1 month without decreasing of appetite or increasing of exercise, so she was recommend to have some protein supplement and follow up weekly. Three weeks later she represented generalized pruritus, blood serum test showed elevated serum transaminases and total bile acids, ALT 146U/L, AST 121U/L, TBA 16.5umol/1, serum albumin was $28 \mathrm{~g} / 1$, luckily ultrasound showed good development her both fetus, so we decided to let her continue following up in outpatient room. Since Intrahepatic Cholestasis Pregnancy (ICP) was suspected on the basis of elevated bile acid and her symptom, S-adenosyl-L-methionine (SAMe) andursodeoxycholic acid (UDCA) were added to her treatment accordingly. One week later her serum albumin drop to $26 \mathrm{~g} / 1$ with TBA increasing to $40 \mathrm{umol} / 1$ (Figure 3), proteinuria remained negative, electrocardiography showed broadening of pulmonary artery, considering the high possibility of recurrent pre-eclampsia, pulmonary hypertension and sudden fetal death, she was admitted at her $30^{\text {th }}$ week gestation to the obstetrics ward, besides all the 
treatment mentioned above, two dose of $12 \mathrm{mg}$ Intramuscular dexamethasone $24 \mathrm{~h}$ apart was administered for fetal lung maturation, under intensive care and all the supportive treatment, her serum transaminases was improved but her blood pressure increased to $156 / 98 \mathrm{mmHg}$ at $31^{\text {th }}$ week of gestation, proteinuria was $0.16 \mathrm{~g} / 24 \mathrm{~h}$, left ventricular diastolic volume also enlarged compare with the index before, TBA elevated to 66umol/1. With the diagnoses of Pre-eclampsia (severe), Intrahepatic Cholestasis of Pregnancy (severe type), Twin pregnancy, vaginal delivery may take long time and may worse the condition and increase the risks of developing eclampsia and fetal death. cesarean section seemed unavoidable. She gave birth to twin sisters weighting $1840 \mathrm{~g}$ (Apgar score 8-9-10) and 1660g (Apgar score 8-9-10) under a successful cesarean section at $31^{\text {th }}$ week, the new born babies were sent to NICU for further treatment and care. The evolution of the patient during the puerperium was good with the normalization of the biochemical and pruritus disappear. TBA, ALT, AST decreased to 4.5umol/1, 33U/L, 39U/L, blood pressure changed gradually to normal range (Figure 2) and no proteinuria when she was discharged from hospital in the $7^{\text {th }}$ day after her delivery. Although without any severe complications, her two babies stayed in the NICU for 26 days receiving special care. The evolution of the newborns were good with a normal intellectual and psychical development when discharged from hospital.

Her blood pressure trend (table 1 and figure 1, 2), liver function tests (figure 3 and table 2) during her last 6 months' pregnancy and postpartum index are mentioned below:



Figure 1. Antenatal BP trend.

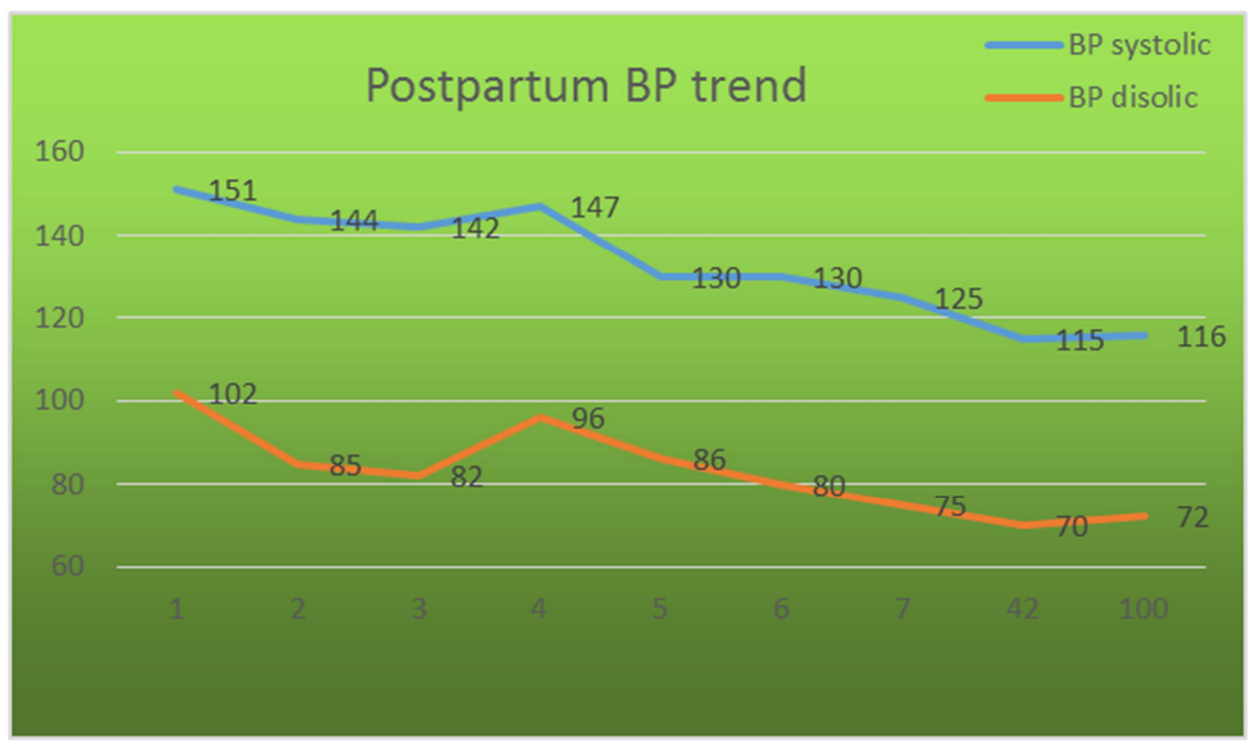

Figure 2. Postpartum BP trend. 
Table 1. Antenatal and postpartum BP index.

\begin{tabular}{llllll}
\hline Weeks (antenatal) & BP Systolic (mmHg) & BP Diastolic (mmHg) & Days (postpartum) & BP Systolic (mmHg) & BP Diastolic (mmHg) \\
\hline $8-12$ & 112 & 73 & 1 & 151 & 102 \\
$13-16$ & 111 & 72 & 2 & 144 & 85 \\
$17-20$ & 115 & 76 & 3 & 142 & 82 \\
$21-24$ & 117 & 79 & 4 & 147 & 96 \\
$25-28$ & 118 & 82 & 6 & 130 & 86 \\
$29-30^{+4}$ & 123 & 88 & 7 & 130 & 80 \\
$30^{+5}$ & 136 & 96 & 425 & 75 \\
$30^{+6}$ & 156 & 98 & 100 & 115 & 72 \\
31 & 151 & & & & \\
\hline
\end{tabular}

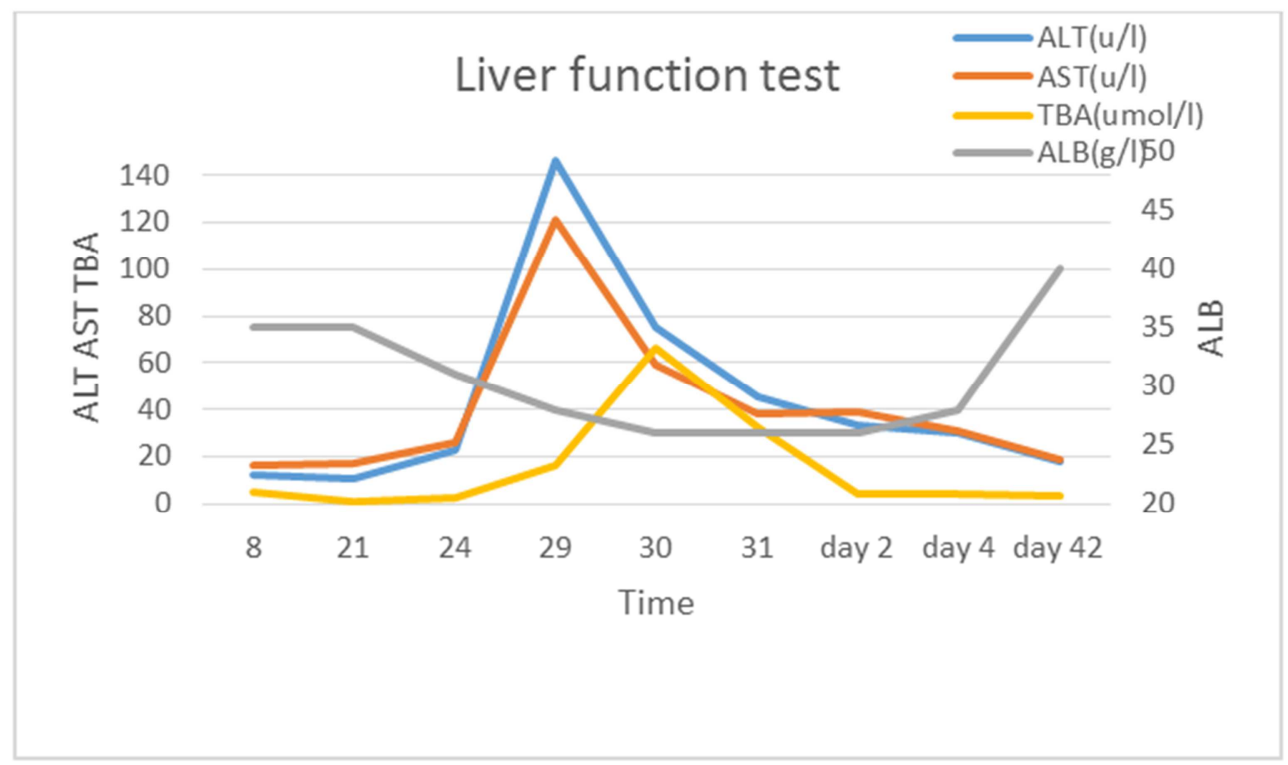

Figure 3. Liver function test.

Table 2. Antenatal and postpartum liver function index.

\begin{tabular}{lllll}
\hline Weeks (antenatal) & ALT (u/l) & AST (u/l) & TBA (umol/l) & ALB (g/l) \\
\hline 8 & 12 & 16 & 5.2 & 34.8 \\
21 & 11 & 17 & 1.2 & 34.8 \\
24 & 23 & 26 & 2.7 & 31.3 \\
29 & 146 & 121 & 16.5 & 28.1 \\
30 & 75 & 59 & 39.5 & 26.6 \\
31 & 45 & 38 & 66.2 & 26.6 \\
Days (postpartum) & 33 & 39 & 4.5 & 26.2 \\
2 & 30 & 31 & 3.2 & 28.2 \\
4 & 18 & 19 & 1.5 & 40 \\
42 & & & & \\
\hline
\end{tabular}

Notion: ALT (Alanime Aminotransferase), AST (Aspartate Aminotransferase), TBA (Total Bile Acid), ALB (Serum Albumin),

\section{Discussion}

Pre-eclampsia is characterized by the new onset of hypertension (blood pressure $\geq 140 \mathrm{mmHg}$ systolic or $\geq 90 \mathrm{mmHg}$ diastolic) at or after 20 weeks' gestation [8]. A review of pre-eclampsia studies in developing countries found prevalence ranging from $1.8 \%$ to $16.7 \%$ [9]. It is a major obstetric problem worldwide that causes significant maternal and neonatal morbidity and mortality [10]. Several studies, including a systematic review and a meta-analysis, have reported an increased risk of pre-eclampsia in pregnancies conceived by in vitro fertilization (IVF) [11-14]. Those patients underwent ART always has infertility, such as in our case the patient is suffered from PCOS, patients with PCOS have a higher risk of pre-eclampsia, because PCOS patients generally have a high ovarian response to $\mathrm{FSH}$, and it has been reported that IVF technology may have certain undesirable side effects, such as OHSS and hypertensive disorders in pregnancy, possibly due to use of drugs and operational intervention [15]. And diminished responsiveness of the ovaries to FSH stimulation in an IVF cycle, which reflects a decreased ovarian reserve, is associated with an increased risk of developing pre-eclampsia in a subsequent pregnancy [16]. Many research has also reported that women with a history of pre-eclampsia have a higher risk of the development of pre-eclampsia in subsequent pregnancies 
[17-22]. It is reported that a history of previous pre-eclampsia is a known risk factor for a new event in a future pregnancy, with recurrence rate varying from less than $10 \%$ to $65 \%$, depending on the population or methodology considered [23]. For severe preeclamptic women in an initial pregnancy, recurrence rates for any type of pre-eclampsia are very high, approaching $50 \%$ in some studies [24]. Patients who conceived through IVF usually had an infertility history, and were extremely nervous, this nervousness may lead to anxiety or depression during pregnancy [25]. The stress experienced by patients may increase resistance of the uterine artery, which is one of the factors leading to pre-eclampsia [26]. Conceiving twice ART and previous pre-eclampsia make our patient extremely anxious than other pregnant women and these may lead to the adverse maternal and neonatal outcome to her. So here comes to our question, our patient has at least two obvious high risks of recurrent pre-eclampsia( PCOS and pre-eclampsia history), although we had done what we could to prevent the recurrence, but according to the figures mentioned above we found the rapidly increasing of $\mathrm{BP}$ and decreasing of liver function at her $31^{\text {th }}$ week of gestation, can the risk decrease if she do fetus reducing in the first trimester of her pregnancy? Or if we transplant just one embryo to those patients who are having IVF and have high risks of pre-eclampsia like our patient, will the recurrent pre-eclampsia incidence decrease? As is well known, Multiple pregnancies are more likely to be associated with pregnancy-induced hypertension [27], Morris $\mathrm{R}$ KP reported that pregnancy outcome rates have improved following selective fetal reduction as experience with techniques have improved and the number of very high order pregnancies has reduced [28]. While Chong-U Cheang has done such research, in his result, he noted that the fetal reduction group was associated with a higher incidence of extreme prematurity, and lower birth weight than the non reduced group [29]. Gelbaya TA suggests that a policy of selective single ET is a logical approach to reduce the incidence of multiple pregnancies after IVF, particularly in young women with good prognosis. There is evidence that in women $<36$ years old, a policy of eSET of cleavage-stage embryos reduces the likelihood of multiple birth by 17 times compared with double ET, with a reduction in live birth likelihood by 1.5 times [30]. But another obvious problem arises, many patients are reluctant to have an elective singleton-embryo transfer(eSET) due to a decreased success rate with less embryos transferred. Amy M. Lee states in his recent article that the voluntary transfer of a single high quality embryo, elective single embryo transfer (eSET), has significantly reduced multiple gestation rates and maximized the rate of singleton pregnancy without compromising overall success rates, he suggests that reproductive medicine should aim for the gold standard IVF outcome to be a singleton term live birth pregnancy with eSET. he claims the future success of ART lies in elective single transfer, the power of one [31].

\section{Conclusion}

The mechanism of hypertensive disorder complicating pregnancy remains to be unclear, some high risks is reported to be related to the high incidence of hypertension. while it is vital importance for the physicians to recognize those high risks group and give preconception counseling or antenatal advice to prevent from pre-eclampsia. With the wider using of the ART and the higher requirements of the patients, comprehensive assessment of physical fitness before conception seems to be very essential. the benefit and risk should be clearly informed to the couples who are ready to have ART specially with high risk factors or pre-eclampsia history. The indications of ART should be strictly controlled and the numbers of embryo implanted have to bring the ultimate benefit to the maternal and fetal health. In conclusion It is never easy for all the obstetricians to specify the best management in a woman with a history of pre-eclampsia in a previous pregnancy. let alone those patients who also have underwent IVF-ET. In this case we applied preconception counseling, psychological intervention, lifestyle intervention, medication (Anti-hypertensive drugs, Low wight molecular heparin, Aspirin, Calcium supplement), patient's self management and surveillance, the pregnancy outcome is little far away from what we have expected. There are no magical interventions to prevent recurrence and promote the maternal and neonatal outcome, all the patient's risk vary from each other, so every single person must be considered individually, which brings different tasks to us. In brief we need to have further research and have good communication with reproductive physicians as well to find a better way. it seems a long journey to go.

\section{References}

[1] Laganà AS, Favilli A, Triolo O, Granese R, Gerli S (2015) Early serum markers of pre-eclampsia: are we stepping forward? J Matern Fetal Neonatal Med 29: 3019-23.

[2] WHO (2004) The Global Burden of Disease, Geneva.

[3] Brown M A, Magee L A, Kenny L C, et al. The hypertensive disorders of pregnancy: ISSHP classification, diagnosis \& management recommendations for international practice [J]. Pregnancy Hypertension, 2018, 72(1):24-43.

[4] Pastore L M. Assisted reproductive technology and pregnancy outcome.[J]. Digest of the World Core Medical Journals, 2016, 107(4):953-4.

[5] Miyake H, Iwasaki N, Nakai A, Suzuki S and Takeshita T: The influence of assisted reproductive technology on women with pregnancy-induced hypertension: A retrospective study at a Japanese Regional Perinatal Center. J Nippon Med Sch 77: 312-317, 2010.

[6] Miyake H, Iwasaki N, Nakai A, et al. The influence of assisted reproductive technology on women with pregnancy-induced hypertension: a retrospective study at a Japanese Regional Perinatal Center.[J]. Journal of Nippon Medical School = Nippon Ika Daigaku zasshi, 2010, 77(6):312. 
[7] Buzzi P J, Zappacosta M P, Auge L, et al. Beneficial effect of local injury to the endometrium in intracytoplasmic sperm injection (ICSI) patients with recurrent implantation failure [J]. Fertility \& Sterility, 2014, 102(3):e293-e293.

[8] Redman C W G, Jacobson S, Russell R. Hypertension in Pregnancy $[\mathrm{M}] / /$ de Swiet's Medical Disorders in Obstetric Practice, Fifth Edition. Wiley - Blackwell, 2010:153-181.

[9] Mahande M, Dalveit A, Mmbaga B, et al. 646: The recurrence risk of preeclampsia in subsequent pregnancies in northern Tanzania: a registry-based prospective cohort study [J]. American Journal of Obstetrics \& Gynecology, 2013, 208(1):S273-S273.

[10] Raymond D, Peterson E: A critical review of early-onset and late-onset preeclampsia. Obstet Gynecol Surv 2011, 66(8):497-506.

[11] Jackson RA, Gibson KA, Wu YW, Croughan MS: Perinatal outcomes in singletons following in vitro fertilization: a meta-analysis. Obstet Gynecol 2004, 103(3):551-563.

[12] Shevell T, Malone FD, Vidaver J, Porter TF, Luthy DA, Comstock CH, Hankins GD, Eddleman K, Dolan S, Dugoff L, et al: Assisted reproductive technology and pregnancy outcome. Obstet Gynecol 2005, 106(5 Pt 1):1039-1045.

[13] Chen XK, Wen SW, Bottomley J, Smith GN, Leader A, Walker MC: In vitro fertilization is associated with an increased risk for preeclampsia. Hypertension Pregnancy 2009, 28(1):1-12.

[14] Thomopoulos C, Tsioufis C, Michalopoulou H, Makris T, Papademetriou V, Stefanadis C: Assisted reproductive technology and pregnancy-related hypertensive complications: a systematic review. J Hum Hypertens 2013, 27(3):148-157.

[15] Woldringh G. P-16: Decreased ovarian reserve relates to preeclampsia in IVF/ ICSI pregnancies [J]. Fertility \& Sterility, 2006, 86(3):S134-S1

[16] Myers ER, McCrory DC, Mills AA, Price TM, Swamy GK, Tantibhedhyangkul J, $\mathrm{Wu} \quad \mathrm{JM}$ and Matchar DB: Effectiveness of assisted reproductive technology (ART). Evid Rep Technol Assess (Full Rep) 167: 1-195, 2008.

[17] Makkonen N, Heinonen S, Kirkinen P. Obstetric prognosis in second pregnancy after preeclampsia in first pregnancy. Hypertens Pregnancy 2000; 19:173-81.

[18] Hnat MD, Sibai BM, Caritis S, et al. Perinatal outcome in women with recurrent preeclampsia compared with women who develop preeclampsia as nulliparas. Am J Obstet Gynecol 2002; 186:422-6.
[19] Trogstad L, Skrondal A, Stoltenberg C, Magnus P, Nesheim BI, Eskild A. Recurrence risk of preeclampsia in twin and singleton pregnancies. Am J Med Genet A 2004; 126A:41-5.

[20] Hjartardottir S, Leifsson BG, Geirsson RT, Steinthorsdottir $\mathrm{V}$. Recurrence of hypertensive disorder in second pregnancy. Am J Obstet Gynecol 2006; 194:916-20.

[21] Brown MA, Mackenzie C, Dunsmuir W, et al. Can we predict recurrence of pre-eclampsia or gestational hypertension? BJOG 2007; 114:984-93.

[22] van Rijn BB, Hoeks LB, Bots ML, Franx A, Bruinse HW. Outcomes of subsequent pregnancy after first pregnancy with early-onset preeclampsia. Am J Obstet Gynecol 2006; 195:723-8.

[23] Costa M L. Preeclampsia: Reflections on How to Counsel About Preventing Recurrence [J]. Journal of Obstetrics \& Gynaecology Canada, 2015, 37(10):887-893.

[24] Iii G A D, Belfort M A, Smulian J C. Preeclampsia Recurrence and Prevention [J]. Seminars in Perinatology, 2007, 31(3):135-141

[25] Xiong F, Hu L, Zhang Y, et al. Correlation of hypertensive disorders in pregnancy with procedures ofin vitrofertilization and pregnancy outcomes:[J]. Experimental $\&$ Therapeutic Medicine, 2017, 14(6):5405-5410.

[26] Kurki T, Hiilesmaa V, Raitasalo R, et al. Depression and anxiety in early pregnancy and risk of preeclampsia $[\mathrm{J}]$. Obstetrics \& Gynecology, 2000, 95(4):487-490.

[27] Sebire NJ, Jolly M, Harris J, Nicolaides KH, Regan L. Risks of obstetric complications in multiple pregnancies: an analysis of more than 400,000 pregnancies in the UK. Prenat Neonatal Med 2001; 6:89-94.

[28] Morris R K, Kilby M D. Multi - fetal Pregnancy Reduction after ART [M]// Assisted Reproduction Techniques: Challenges and Management Options. Wiley - Blackwell, 2012:400-404

[29] Cheang C U, Huang L S, Lee T H, et al. A comparison of the outcomes between twin and reduced twin pregnancies produced through assisted reproduction.[J]. Fertility \& Sterility, 2007, 88(1):47-52.

[30] Gelbaya TA, Tsoumpou I, Nardo LG. The likelihood of live birth and multiple births after single versus double embryo transfer at the cleavage stage:a systematic review and meta-analysis. Fertil Steril 2010; 94:936-45.

[31] Lee A M, Connell M T, Csokmay J M, et al. Elective single embryo transfer- the power of one $[\mathrm{J}]$. Contraception \& Reproductive Medicine, 2016, 1(1):11. 